\title{
Miranda
}

Revue pluridisciplinaire du monde anglophone /

Multidisciplinary peer-reviewed journal on the English-

speaking world

$18 \mid 2019$

Guerre en poésie, poésie en guerre

\section{Catherine Bernard, Matière à réflexion. Du corps politique dans la littérature et les arts visuels britanniques contemporains.}

\section{Françoise Baillet}

\section{OpenEdition}

\section{Journals}

Édition électronique

URL : http://journals.openedition.org/miranda/17665

DOI : 10.4000/miranda. 17665

ISSN : 2108-6559

\section{Éditeur}

Université Toulouse - Jean Jaurès

\section{Référence électronique}

Françoise Baillet, «Catherine Bernard, Matière à réflexion. Du corps politique dans la littérature et les arts visuels britanniques contemporains. », Miranda [En ligne], 18 | 2019, mis en ligne le 16 avril 2019, consulté le 16 février 2021. URL : http://journals.openedition.org/miranda/17665 ; DOI : https:// doi.org/10.4000/miranda.17665

Ce document a été généré automatiquement le 16 février 2021.

\section{cc) (1)}

Miranda is licensed under a Creative Commons Attribution-NonCommercial-NoDerivatives 4.0

International License. 


\section{Catherine Bernard, Matière à} réflexion. Du corps politique dans la littérature et les arts visuels britanniques contemporains.

Françoise Baillet

\section{Matière à réflexion}

Du corps politique dans la littérature et les arts visuels britanniques contemporains

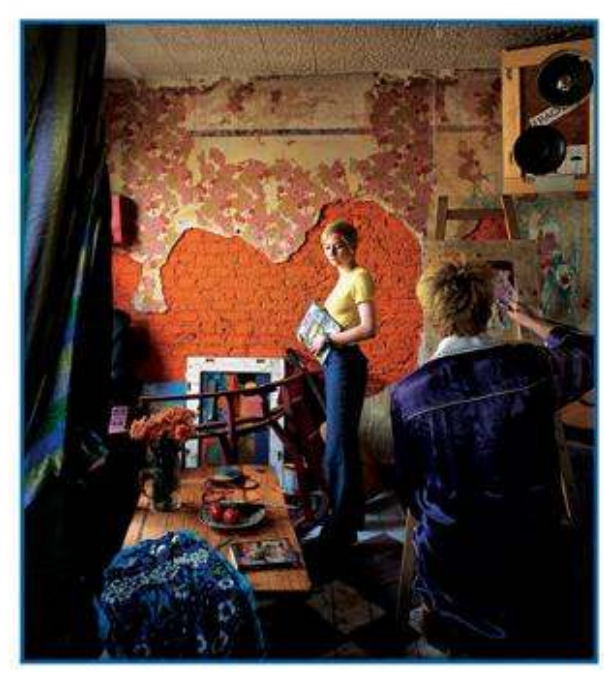

Catherine Bernard

Dans sa présentation de la revue Les Temps Modernes, qui parait chez Gallimard à partir d'octobre 1945, Jean-Paul Sartre revendique l'engagement de l'écrivain dans son époque : «Puisque l'écrivain n'a aucun moyen de s'évader, » écrit-il, « nous voulons 
qu'il embrasse étroitement son époque; elle est sa chance unique, elle s'est faite pour lui et il est fait pour elle $»^{1}$. C'est cette rencontre, jadis reniée par le modernisme, qui se trouve au cœur de Matière à réflexion, publié en 2018 par Catherine Bernard aux Presses Universitaires de Paris Sorbonne. Sous-titré Du corps politique dans la littérature et les arts visuels britanniques contemporains, cet ouvrage soigné de 362 pages s'attache à examiner la manière dont la représentation artistique met en forme et réfléchit le politique dans un contexte de crise. Spécialiste de la modernité esthétique et auteur, entre autres, d'une édition critique d'essais de Virginia Woolf, Catherine Bernard se penche en particulier sur les effets de la dérégulation économique sur les pratiques esthétiques. Dans un contexte de déréliction du tissu social exacerbé par les effets du libéralisme économique thatchérien, écrivains et artistes, nous dit-elle, « retournent le sentiment de crise contre lui-même pour en faire un moment d'intellection historique » (19). Ainsi c'est bien une renaissance qu'évoque ici Catherine Bernard, pour qui le concept de représentation, loin de l'épuisement qu'on a pu lui prêter, se trouve "réincarné et revitalisé » (303) par la création littéraire et artistique britannique contemporaine. «Plus que jamais, » nous dit-elle, « la littérature et les arts plastiques britanniques se veulent comptables de ce qui nous délie et nous lie » (21).

2 Ouvrage érudit, dont le propos s'appuie sur des sources nombreuses, variées et maîtrisées, tant du point de vue du corpus primaire que de l'appareil critique qui permet de l'appréhender, Matière à réflexion propose ainsi un parcours analytique optimiste au terme duquel l'imbrication entre art et société se trouve réaffirmée: "l'art et l'écriture pensent le monde, car ils sont à son contact» (304). Certes, ce constat n'est pas nouveau. Espace de production et de mise en scène, la représentation constitue l'un des rouages essentiels de la culture dont elle construit, par des moyens visuels autant que verbaux, certains positionnements idéologiques. Roland Barthes rappelle que l'image « est un objet travaillé, choisi, composé, construit, traité selon des normes professionnelles, esthétiques ou idéologiques, qui sont autant de facteurs de connotation $»^{2}$. Elle n'est pas seulement perçue, elle est "relue, elle est lue, rattachée plus ou moins consciemment, par le public qui la consomme, à une réserve traditionnelle de signes $»^{3}$. Ainsi, loin de n'être que spectateur de l'image, celui qui la regarde en fait partie intégrante, projetant sur celle-ci des constructions et valeurs "toujours élaborés par une société et une histoire définies »" Mais par-delà cette présence de l'artiste dans son époque, l'originalité de l'approche de Catherine Bernard consiste à aborder cet engagement au prisme de la sensation. Dans Matière à réflexion, c'est la matérialité même de l'œuvre, et pas seulement son sujet, qui réfléchit, qui fait réfléchir sur le politique. Charpentée en trois temps - «sonder l'imaginaire malade du monde contemporain » (30) (chapitres 1 et 2), « incarner la mémoire collective » (141) (chapitre 3) et « faire société malgré tout » (chapitres 4 et 5) - la trajectoire analytique de l'ouvrage nous mène de la désagrégation sociale au « bruissement du nous » (286), du démembrement aux « entrelacs complexes du sens et de la sensation » (70).

Les deux premiers chapitres - «La Fin des fins ? Et encore après... ", "Un Art de/en guerre » - s'attachent à analyser la façon dont la fiction britannique contemporaine, celle des années 1980 en particulier, s'est repensée en écriture de la crise. Convoquant une large palette d'auteurs parmi lesquels Graham Swift, Maggie Gee, Julian Barnes et Jeanette Winterson, Catherine Bernard voit dans la dislocation de la structure narrative à l'œuvre dans une sélection de romans une stratégie visant à « résiste[r] poétiquement à toute clôture signifiante » (36). La " fin de l'art ", identifiée par Hegel dans ce moment où celui-ci devient « une simple démonstration de virtuosité, une pure ostentation de 
soi $»^{5}$ et mise en œuvre, nous dit Bernard, sous la forme d'une vision allégorique exacerbée, devient ici une forme de résistance à la fin du monde, à la fin d'un monde. Fracturer la forme pour ne pas disparaitre. Dans cette "dramaturgie du politique " (88), le corps - physique, social - devient lisible. Et tandis que les romans de guerre donnent à voir la "nudité même des rapports de force » (89), formule empruntée à Foucault, l'image, celle de McQueen dans Hunger (2008), par exemple, "fait de la matière organique le vecteur d'une réflexion politique sur la citoyenneté » (112). Le partage du sensible s'inscrit ici dans une perspective from below, où focalisation interne et corps subalternes meurtris révèlent les mécanismes du trauma (95).

4 C'est précisément cette conscience aiguë d'une crise de la représentation qui, dans le chapitre suivant, permet l'élaboration d'une pensée critique salutaire. Pivot de l'ouvrage, le chapitre 3 voit dans la mémoire collective et la façon dont celle-ci est incorporée dans l'expérience visuelle et textuelle la source d'un renouveau esthétique et politique. "Les traces du passé - traces historiques, intertextuelles, esthétiques nous reviennent. Elles survivent et surviennent encore. Elles insistent» (141), nous dit Catherine Bernard. Et si la peau humaine, celle que Mona Hatoum ou Jenny Saville nous donnent à voir dans des œuvres telles que Measures of Distance (1988) ou Closed Contact (1995-1996), porte les traces de son histoire, l'intertextualité, celle de Peter Ackroyd dans English Music (1992) ou The English Ghost (2010) fait littéralement écho au passé, rappelant que « l'écriture s'accomplit comme survivance plus qu'elle n'advient » (143).

5 Le corps - charnel, textuel - ainsi investi comme lieu de reconstruction; ne reste alors à l'art qu'à repenser, à réinventer la communauté. C'est là l'objet des deux derniers chapitres, "Corps habités/corps habitant " et "Multitudes et communauté », où, explique Catherine Bernard, la notion de représentation esthétique et politique se voit court-circuitée au profit d'expressions, de présents "labiles, fugaces, insaisissables » (186). Au « morcellement qui les tenait indéfiniment à distance de leur propre temps, » il s'agit ainsi pour les humbles et déclassés qui peuplent les films de Ken Loach comme les récits d'Irvine Welsh, de Zadie Smith ou de Caryl Phillips, d'opposer ce que Jacques Rancière nomme "une fragmentation qui leur en rend la maîtrise et construit un possible nouveau $»{ }^{6}$ Reconquérir le temps, c'est reconquérir le visible. C'est par une écriture de la colère autant que par la multiplicité des voix narratives que s'opère cette reconquête, toujours en devenir: "Le commun reste, plus que jamais, à imaginer » (310).

6 Solidement nourrie par la production littéraire, artistique et théorique contemporaine, ainsi qu'en attestent une bibliographie de 37 pages qui fait la part belle aux textes critiques et un index fourni et subdivisé en trois catégories (écrivains, artistes et cinéastes, philosophes et critiques), cette étude constitue de toute évidence une référence essentielle pour les spécialistes des questions abordées. Peut-être s'adresse-telle moins directement à un public plus large - celui, par exemple, des anglicistes ou des amateurs de littérature britannique - en raison de la technicité de la terminologie utilisée par endroits, qui peut parfois faire écran à la démonstration. Comme si Catherine Bernard elle-même hésitait à entrer en contact avec cette matière qui nourrit son propos, comme si à la peau meurtrie de son corpus elle opposait un argumentaire lisse, policé : une peau lissée. Et tandis que son lecteur est d'emblée saisi par la puissance de certaines œuvres citées, il peut, en fonction de sa formation théorique, se trouver dérouté par la formulation quelque peu absconse de leur analyse (116). Restent la finesse, la précision et le ciselé de Matière à réflexion. Reste également 
l'apport considérable de cette étude à la confluence de la littérature, de l'esthétique et de la philosophie, qui donne la mesure des liens unissant art, société, fiction et politique.

\section{NOTES}

1. Jean-Paul Sartre, Les Temps modernes 1, octobre 1945, 20.

2. Roland Barthes, Euvres complètes, vol. 1, Paris: Seuil, 1993, 940.

3. Ibid., 940.

4. Ibid., 946.

5. Jacques Rancière, Les Temps modernes. Art, temps, politique. Paris : La Fabrique, 2018, 55.

6. Ibid., 37.

\section{INDEX}

Mots-clés : littérature, arts visuels, cinéma, peinture, politique britannique 20ème siècle, Thatchérisme

Keywords : literature, visual arts, cinema, painting, 20th c. British politics, Thatcherism

\section{AUTEURS}

\section{FRANÇOISE BAILLET}

Professeur de civilisation britannique/études visuelles.

Université de Caen

francoise.baillet@unicaen.fr 